\title{
Using different types of neural networks in detection the body's readiness for blood donation and determining the value of each of its parameters using genetic algorithm
}

Uso de diferentes tipos de redes neurales en la detección de la preparación del cuerpo para la donación de sangre y determinando el valor de cada uno de sus parámetros utilizando el algoritmo genético

Author:

Zahra Jafari ${ }^{1 *}$

Asma Mahdavi Yousefí ${ }^{2}$

Saman Rajabi ${ }^{3}$

\section{SCIENTIFIC RESEARCH}

How to cite this paper:

Jafari Z., Mahdavi Yousefi A. and Rajabi S., Using different types of neural networks in detection the body's readiness for blood donation and determining the value of each of its parameters using genetic algorithm, Iran. Innovaciencia 2020; 8 (1): 1-10. DOI: http://dx.doi. org/10.15649/2346075X.998

\section{ABSTRACT}

Blood transfusion is of great importance in the medical field, as blood donation centers are responsible for collecting and distributing blood and blood products. Artificial neural network is a data processing system getting ideas from the human brain and designs a data structure that acts like a neuron using programming science and by creating a network between these neurons and combining large amounts of data with smart algorithms and their rapid processing, the network is trained. In this study, data is extracted from the blood transfusion service center and the perceptron neural network, RBF neural network, Fisher's discrimination ratio and genetic algorithm were examined, and finally the highest possible accuracy from the neural network was achieved.

Reception date:

Received: 01 May 2020

Accepted: 01 August 2020

Published: 01 December 2020

Keywords:

Perceptron neural network, RBF neural network, blood transfusion, Fisher's discrimination ratio, genetic algorithm.

1* Department of Medical Engineering, Faculty of Electrical Engineering, Tabriz Branch, Islamic Azad University, Tabriz, Iran. Corresponding author: stu.z.jafari@iaut.ac.ir

2 Department of Medical Engineering, Faculty of Electrical Engineering, Tabriz Branch, Islamic Azad University, Tabriz, Iran

3 Assistant Professor of Electrical Engineering Department, Seraj Higher Education Institute, Tabriz, Iran 


\section{INTRODUCTION}

Blood donation means the transfusion of a predetermined amount of blood from healthy people in blood transfusion centers. After screening, the blood is donated by a blood bank to patients in need of blood or used to make blood products. Individuals who had undergone surgery, or have had an accident, and have lost a lot of blood, or people who have a specific condition such as thalassemia or leukemia, need blood. Also, individuals with certain diseases, such as hepatitis or AIDS, cannot donate blood because these diseases are transmitted through blood. [1] The database contains 748 samples that have been investigated. In this data bank, each sample contains a variable and the given features are shown in Table 1. Variable 1 indicates blood donation and variable 2 indicates non-donation of blood by an individual.

Table 1: Features related to database

\begin{tabular}{lll}
\hline The last months since the last donation & $\mathrm{R}$ & 1 \\
Total number of donations & $\mathrm{F}$ & 2 \\
The total donated blood in c.c & $\mathrm{M}$ & 3 \\
The last months since the first donation & $\mathrm{T}$ & 4 \\
\hline
\end{tabular}

\section{METHOD}

In this study, the accuracy of neural networks is calculated using a simple perceptron neural network and a RBF neural network. Then, some coefficients are obtained using Fisher's discrimination ratio and genetic algorithm to minimize the number of features and accelerate the time and speed and improve the accuracy of the neural network.

\subsection{Neural network}

Artificial neural network is a data processing system that takes ideas from the human brain and delegates the processing of data to small and large processors so that they can solve a problem in a continuous and parallel manner. In these networks, using programming knowledge, a data structure is designed that can act like a neuron. This data structure is called neuron. By creating a network between these neurons and applying an educational algorithm to it, the network is trained. [2] A neural network consists of the constituent components of layers and weights. Network behavior also depends on the communication between members. In general, there are three types of input, hidden and output neural layers in neural networks. In the neural network, neurons have two active states (on or 1 ) and inactive (off or 0 ), and each synapse has one weight [3 and 4].

\subsubsection{Perceptron Neural Network}

One type of neural network is the perceptron neural network, which is available in the form of singlelayer and multi-layer perceptron network. Perceptron neural networks are classified as feedforward neural networks. Single-layer perceptron can only classify separate linear problems, and for more complex problems we use more layers. Multi-layer feedforward networks consist of one or more intermediate layers. The multilayer perceptron is a completely interconnected network because each neuron in one layer is connected to all the neurons in the next layer. If some of these connections do not exist, the network is an incomplete connected network. Figure 1 shows an example of a perceptron neural network. [5] 


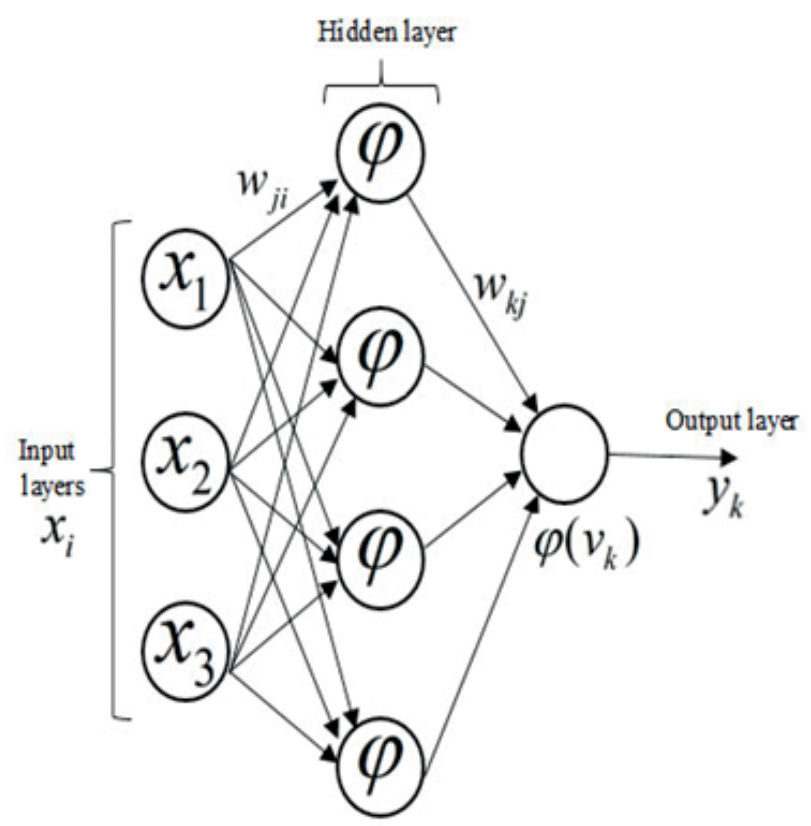

Figure 1: An example of multilayer perceptron neural network

The input-output equations of the $\mathrm{k}^{\text {th }}$ neuron are as follows:

$$
\begin{aligned}
& Y_{k}=\varphi\left(v_{k}\right) \\
& v_{k}=\sum_{j=1}^{n} W_{j k} x_{j}
\end{aligned}
$$

Where $x_{1}, x_{2}, x_{3}, \ldots, x_{n}$ are input signals. $u_{k}$ is the sum output. $W_{k 1}, W_{k 2}, W_{k 3}, \ldots, W_{k n}$ are weights of neurons. The final output of the MLP network with a hidden layer is equal to:

$$
Y=\sum_{k=1}^{N} W_{k} Y_{k}
$$

In other words:

$$
Y_{k}=\varphi\left(\sum_{j=1}^{n} W_{j k} x_{j}\right)
$$

The active function used in the hidden layer is usually nonlinear, and the transform function in the output layer can be linear or nonlinear.

Perceptron algorithm is a repetitive algorithm, in which the weight vector and bias are quantified, and then at each step, the algorithm changes the weight and bias values according to the points that are not properly classified so that these points are classified accurately. Categorize. If the given points are not separated in a linear manner, the perceptron algorithm is not finished, but if the linear points are separated, the algorithm will end in a finite number of steps. 
First, a single-layer perceptron neural network is designed for all features as shown in Figure 2.

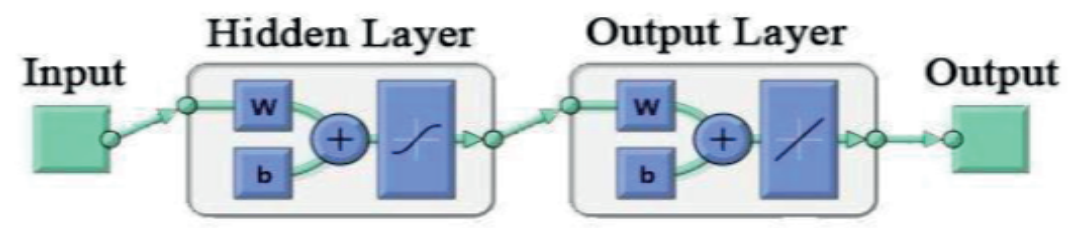

Figure 2: Single-layer perceptron neural network for all features

By changing all possible parameters in a perceptron neural network, including the number of hidden layers, the number of neurons in each layer, and the transform functions of each neuron, the best possible accuracy for the neural network is obtained as a network shown in Figure 3 with 60.52\% accuracy.

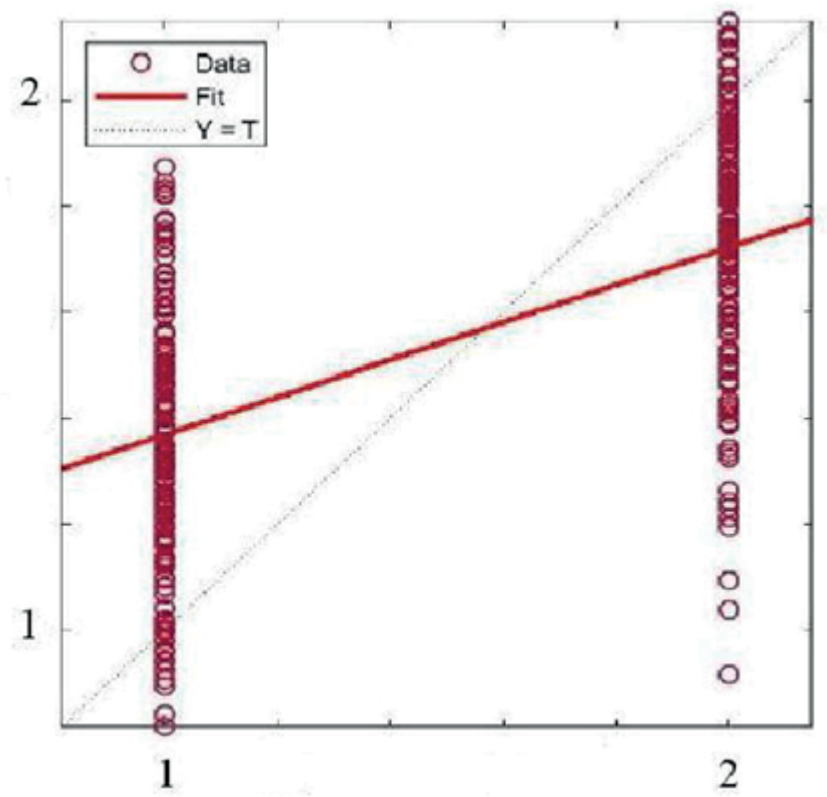

Figure 3: Single-layer perceptron neural network regression with all features and $60.52 \%$ accuracy

The points outside the dash- line indicate an error in classification with the neural network as shown in Figure 3.

\subsection{RBF neural network}

The artificial neural network RBF consists of neural cells and uses radial basis functions as activity functions. The output in the RBF neural network is a linear combination of radial basis functions for input parameters and neurons. Similar to the pattern The RBF neural network is shown in Figure 4. used in MLP neural networks, there is another type of neural network in which processor units focus on a specific situation in terms of processing. This concentration is modeled through radial basis functions (RBF). In general, the RBF neural network is not different from the MLP network, and only the type of processing at the input of the neurons is different. However, RBF networks often have faster learning and preparation processes. These networks are used in approximation, time series prediction, classification and system control functions [6]. 


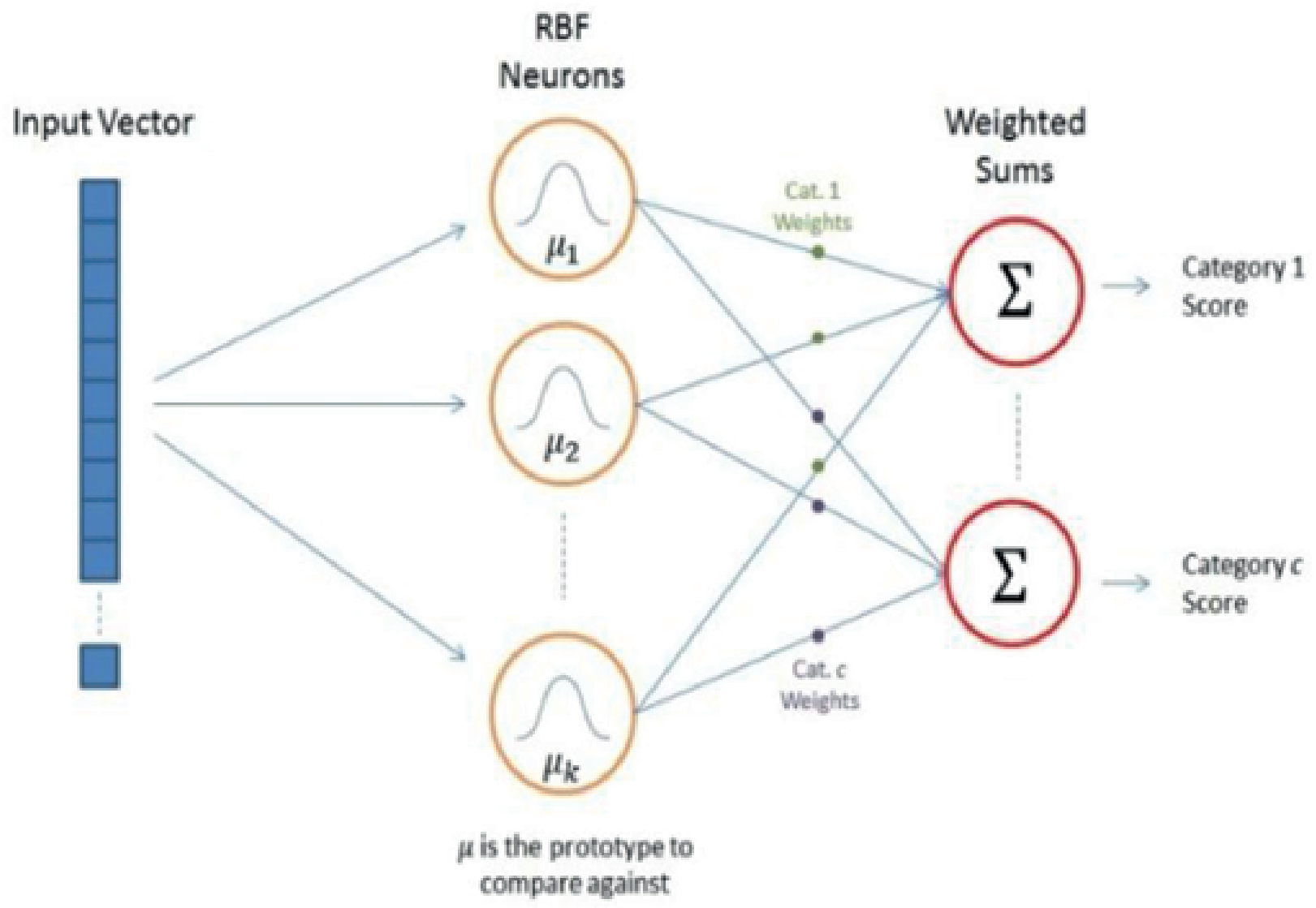

Figure 4: Neural network of radial basis function [7]

A RBF neural network is simulated as shown in Figure 5. The change function used for the hidden layer is the Gaussian function, which is classified in radial basis functions.

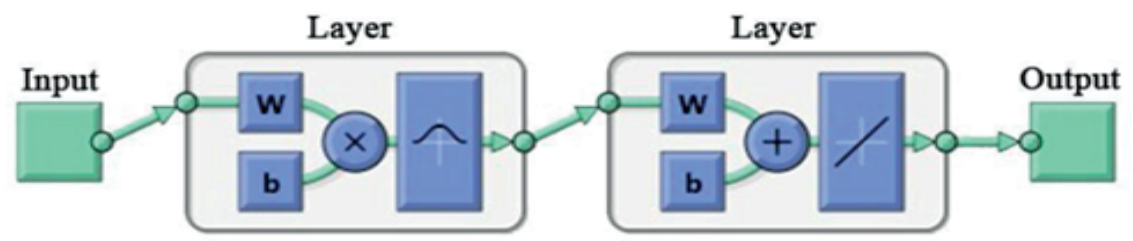

Figure 5: RBF neural network designed for all features

RBF neural network is selected with 500 designed radial functions and $93.21 \%$ accuracy is achieved. It can be seen that the classification accuracy of the RBF neural network has improved significantly com- pared to the classification of the perceptron neural network $(60.52 \%)$. The regression of the RBF neural network is shown in Figure 6. 


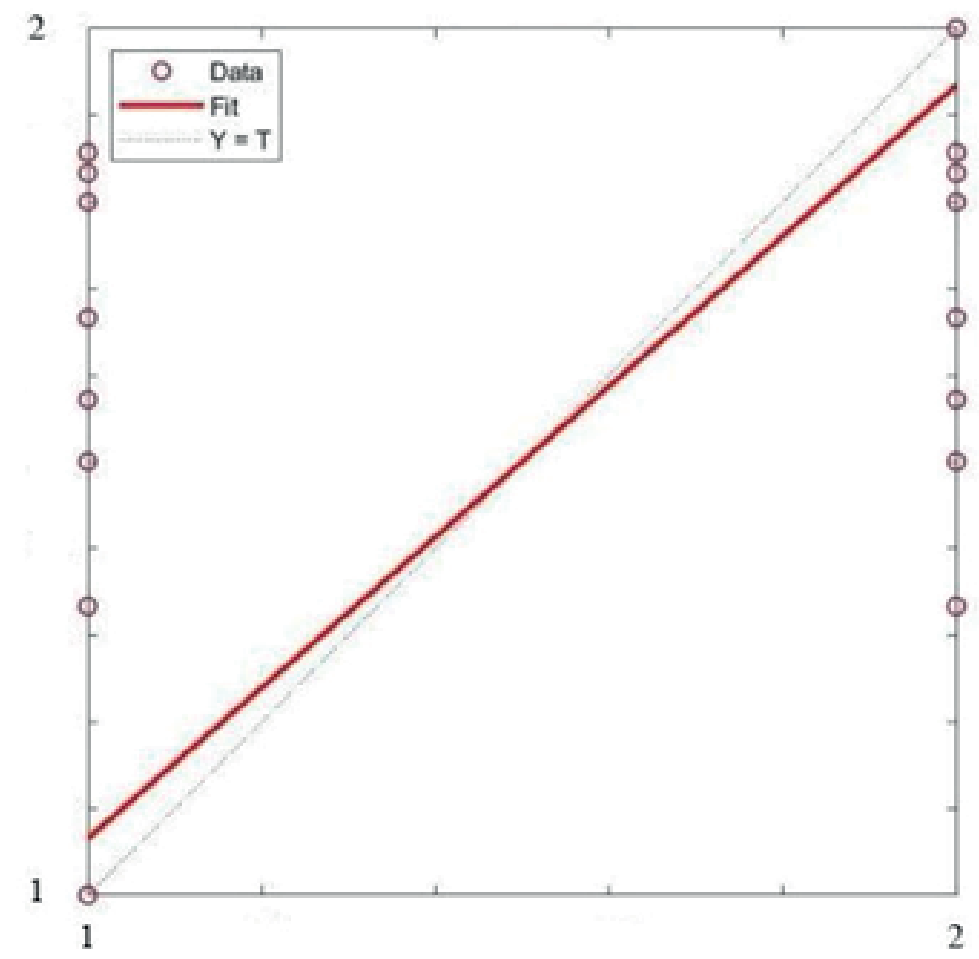

Figure 6: RBF neural network regression with all features and 93.21\% accuracy

The values of radial basis functions will have a considerable effect on the CCR classifier, so it has been investigated and its effect on accuracy is as shown in Figure 7.

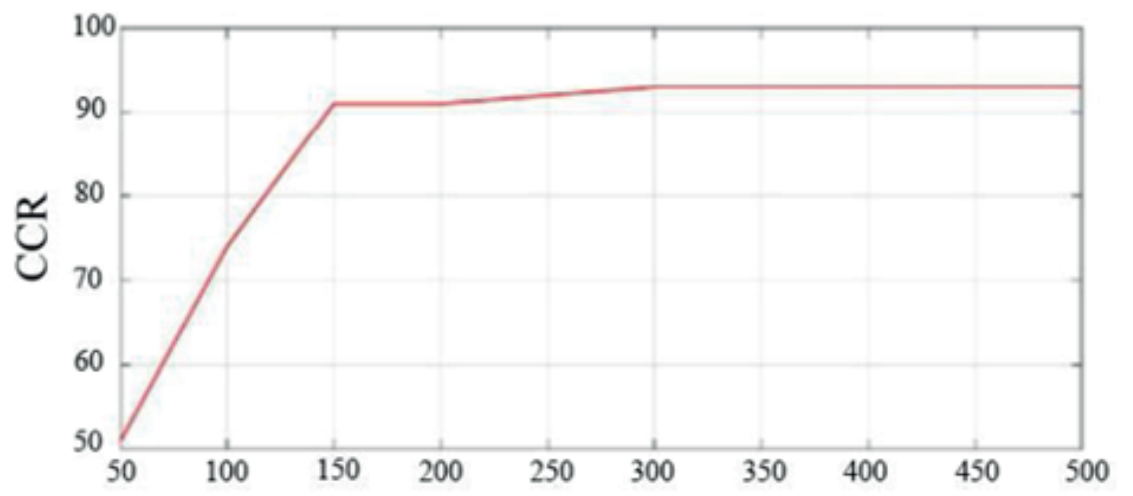

Number of radial based functions

Figure 7: The effect of the number of radial basis functions on the accuracy of the radial neural network

\subsection{Fisher's discrimination ratio}

Assuming a Gaussian or quasi-Gaussian distribution for the remaining features, it can be assumed that if the mean difference between the features of the two classes is greater and the variance of the features of each class is smaller, the discriminability of these two classes will be better. This is shown in Figure 8. 


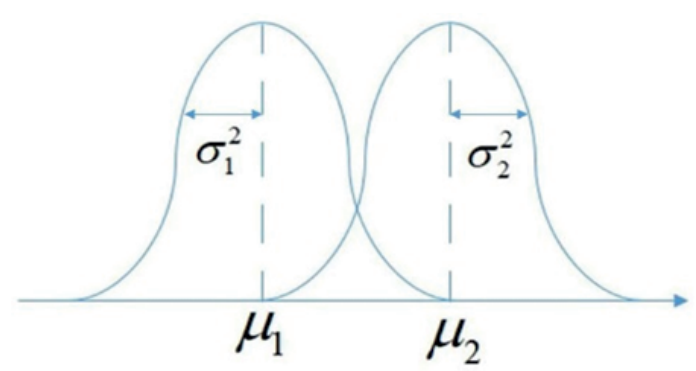

Figure 8: Diagram of detecting the discriminability of two classes

As it was mentioned, the FDR term for a two-class problem is defined as follows:

$F D R=\frac{\left(\mu_{1}-\mu_{2}\right)^{2}}{\sigma_{1}^{2}+\sigma_{2}^{2}}$

Where we have:

$$
\mu=\left(\mu_{1}+\mu_{2}\right) / 2
$$

If the mean difference is greater and the variances are lower, the FDR will increase. Therefore, increasing FDR means increasing class separation and thus increases classification accuracy.

\subsection{Genetic algorithm}

Genetic algorithm can be called a general search method that imitates the rules of natural biological evolution. The genetic algorithm is one of the subsets of evolutionary algorithms, which is directly related to the subject of artificial intelligence. In fact, genetic algorithm is one of the subsets of artificial intelligence. The genetic algorithm applies the law of survival of the fittest to a series of solutions to the problem in the hope of achieve better solutions. In each generation, with the help of a selection process consistent with the value of the answers and the reproduction of the selected solutions achieves better approximations of the final answer by the operators that have been imitated from natural genetics. This process makes the new generations more adaptable to the conditions of the problem.

Genetic algorithm, as an optimization computational algorithm, effectively searches different areas of the solution space, considering a set of points in the solution space, in each repetition. In the search mechanism, although the objective function is not calculated for all points in the solution space, the process of the algorithm proceeds in such a way that the convergence probability of the solution to the absolute optimal point is higher than its convergence to the local optimal point. [8,9]

The most important operator in the genetic algorithm is the combination operator. Combination is the process by which the older generation of chromosomes mixes and combines to form a new generation of chromosomes. Combination in the genetic algorithm eliminates the dispersion or genetic diversity of the population because it allows the good genes to find each other.

In the combination of features for each of the features of the combination, a parametric weight is considered to obtain the possibility of optimizing (maximizing) the FDR value. For example, to combine $\mathrm{n}$ features, the final weight as $a=\left[a_{0}, a_{1}, \ldots, a_{n}\right]$ is applied to the given features. Then, using genetic optimization algorithm, the values of these coefficients are determined as the given FDR is maximized.

The genetic algorithm method is based on minimizing the objective function, but since the goal is to maximize FDR, the fitness function of the genetic algorithm is defined as follows: 
Fitness.Function $=\frac{1}{F D R}=\frac{\sigma_{1}^{2}+\sigma_{2}^{2}}{\left(\mu_{1}-\mu\right)^{2}+\left(\mu_{2}-\mu\right)^{2}}$

In this study, the features presented in Table 2 are combined with the coefficients shown, and after maximizing the FDR with the genetic algorithm, they become an optimal feature.

Table 2. Combined features

\begin{tabular}{lll}
\hline Feature & & Coefficient \\
\hline The last months since the last donation & $\mathrm{R}$ & $a_{1}$ \\
Total number of donations & $\mathrm{F}$ & $a_{2}$ \\
The total donated blood in c.c & $\mathrm{M}$ & $a_{3}$ \\
The last months since the first donation & $\mathrm{T}$ & $a_{4}$ \\
\hline
\end{tabular}

How to apply the coefficients is as follows:

$$
F D R=\frac{a_{1 \times 4}^{\prime}\left(\mu_{1}-\mu_{2}\right) \times a_{4 \times 1}}{a_{1 \times 4}^{\prime} \times \sigma_{1_{4 \times 4}}^{2} \times a_{4 \times 1}}
$$

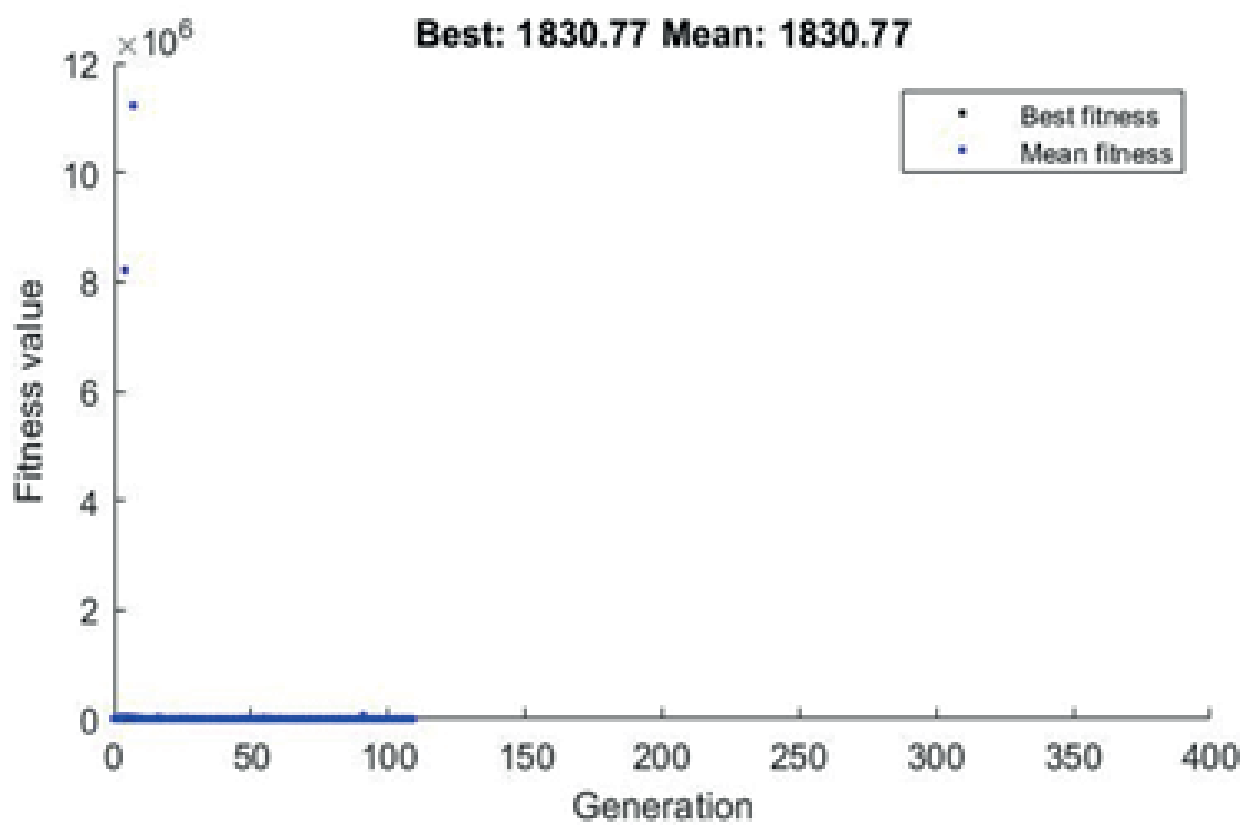

Figure 9: The chart of minimization the fitness function in genetic algorithm 


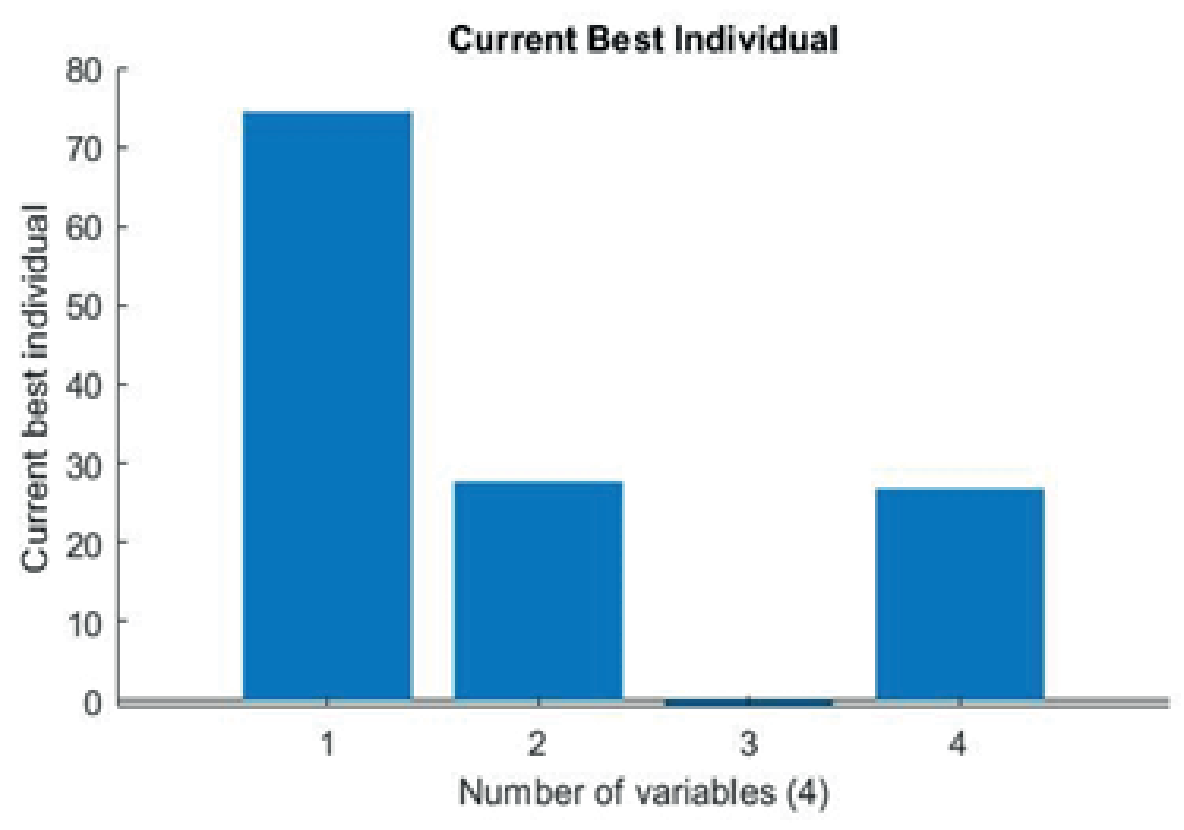

Figure 10: The Chart of optimized coefficient

After optimizing $a_{1}=74.485, a_{2}=27.672 a_{3}=-0.795$ and $a_{4}=26.717$, instead of the features shown in Table 2, the folflowing single feature is replaced:

Combined feature $=\left(a_{1} \times R\right)+\left(a_{2} \times \mathrm{F}\right)+\left(a_{3} \times \mathrm{M}\right)+\left(a_{4} \times \mathrm{T}\right)$

By performing the processing, the number of features is reduced from 4 to 1 . To evaluate the accuracy of the work, again a perceptron neural network optimized according to the figure is designed and with the remaining single-feature, the classification accuracy is reported $100 \%$ as its improved compared to the previous stage neural network with the accuracy $93.21 \%$.

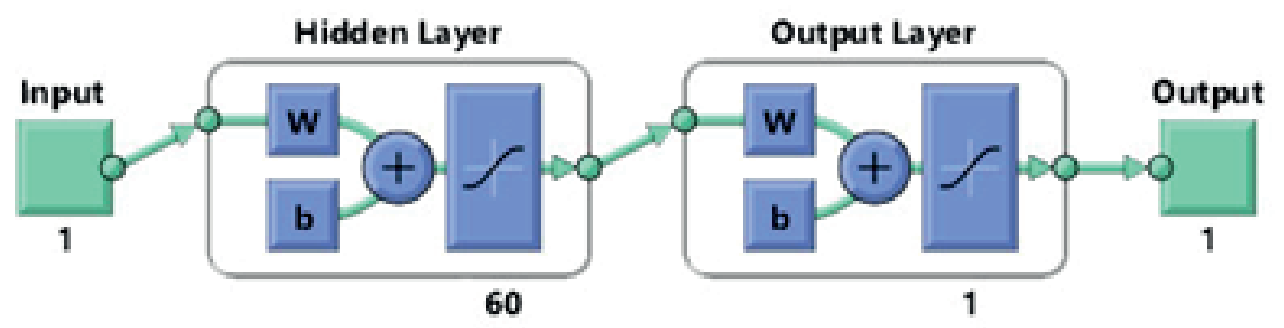

Figure 11: Perceptron neural network with the remaining single feature after combining the features 


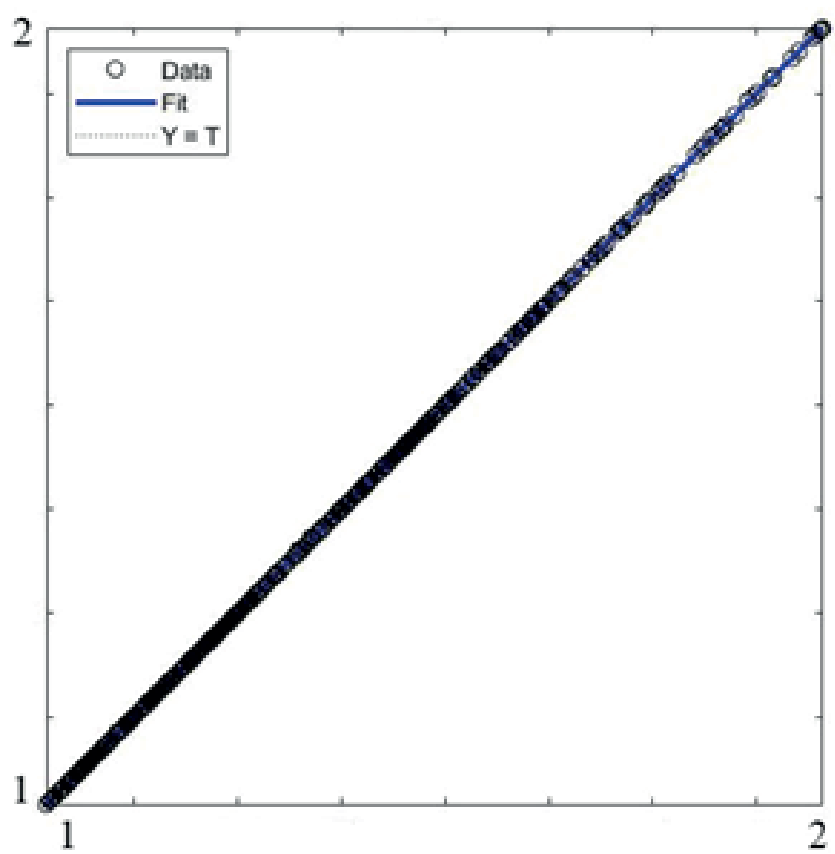

Figure 12: Neural network regression with the remaining single feature after combining the features with $100 \%$ accuracy

\section{CONCLUSION}

Blood transfusion is the injection of blood into the body in case of illness or injury and blood loss. If the body loses healthy blood compounds, blood transfusion can help to provide it again. In this paper, the initial clustering was performed using the Perceptron neural network and the accuracy was achieved $60.52 \%$. Then, with the formation of the RBF neural network, clustering was performed again with the accuracy of $93.21 \%$. Finally, using the Fisher's discrimination ratio and the combination of features, the number of features was minimized to increase the accuracy and speed of the neural network. It is observed that after combining the features, the accuracy of the neural network is increased to $100 \%$.

\section{REFERENCES}

1. American Red Cross Biomedical Services. Archived from the original on 2009-10-20. Retrieved 2009-10-26.

2. R. Beale and T. Jackson, Neural Computing (An Introduction), Adam Ililger (1990).

3. J. Schmidhuber, "Deep Learning in Neural Networks: An Overview”. Neural Networks. 61: 85117. (2015).
4. D.Graupe, principles of artificial neural networks, world scientific publishing co. Pte. LTD., Vol.6, second edition, 2007.

5. U. Orhan, M. Hekim, M. Ozer, "EEG signals classification using the K-means clustering and a multilayer Perceptron neural network model" Expert Systems with Applications 38 (2011) 1347513481.

6. Lowe, D, "Adaptive radial basis function non-linearities and the problem of generalisation", IEE Conf. on Artificial Intelligence and Neural Networks. 1989.

7. C. K. I. Williams, D. Barber "Bayesian Classification with Gaussian Process" IEEE Transactions on Pattern Analysis and Machine Intelligence (Volume: 20, Issue: 12, Dec 1998).

8. Seryasat, Omid Rahmani, and Javad Haddadnia. "Evaluation of a new ensemble learning framework for mass classification in mammograms." Clinical breast cancer 18.3 (2018): e407-e420.

9. J.L.R. Filho, P.C. Treleaven, C. Alippi, Genetic algorithm programming environments, IEEE Comput. 27 (1994) 28-43. 